\title{
Effects of supraphysiological doses of nandrolone decanoate in Achilles tendon of rats
}

\author{
P. Pires Marques ${ }^{1}$, N. Mota Porto ${ }^{2}$, C. Pedrozo Vieira ${ }^{3}$, L. Prado Oliveira4, A. Esteves ${ }^{2}$, \\ W. Costa Rossi Júnior ${ }^{2}, E$. Rosa Pimente ${ }^{1,4}$, F. Da Ré Guerra ${ }^{2}$ \\ 1 Department of medicine, José do Rosário Vellano University (UNIFENAS), Alfenas, Brazil. Rua Gabriel Monteiro \\ da Silva, 700, 37130-000, Alfenas, MG, Brazil \\ 2 Department of Anatomy, Institute of Biomedical Science, Federal University of Alfenas - UNIFAL-MG, Alfenas, \\ MG, Brazil. Rua Gabriel Monteiro da Silva, 700, 37130-000, Alfenas, MG, Brazil \\ 3 Department of Pharmacology Faculty of Medical Sciences, The University of Alabama at Birmingham. \\ Department of ophthalmology, 1720 2nd Ave South, Birmingham, AL 35294 \\ 4 Department of Anatomy, Cell Biology and Physiology and Biophysics, Institute of Biology, Cidade Universitária \\ Zeferino Vaz - Barão Geraldo - Rua Monteiro Lobato, 255, 13083-970, Campinas, SP, Brazil
}

\section{CORRESPONDING AUTHOR: \\ Flávia Da Ré Guerra \\ Institute of Biomedical Sciences \\ Department of Anatomy, \\ Federal University of Alfenas \\ Alfenas, Brazil \\ E-mail: unifal.flavia@unifenas.br \\ Phone: +55 35 3701-9564}

DOI:

10.32098/mltj.02.2019.15

LEVEL OF EVIDENCE:

$2 b$

\begin{abstract}
SUMMARY
Background. Anabolic androgenic steroids (AAS) are a group of natural compounds derived from testosterone. It promotes a rapid increase in muscle mass as well as an individual's strength, thus overloading the locomotor system. Injuries, such as tearing of ligaments and tendons and damaged cartilage, are described among users of these steroids. Methods. To study the effects of nandrolone decanoate (ND) on tendons, male adult Wistar rats were distributed in G1 - control group; G2 - sedentary animals treated with ND; G3 - trained animals; G4 - trained animals treated with DN. After 8 weeks of treatment, the animals were submitted to euthanasia and the tendon was removed for further analyzes. Results. Non-collagenous protein quantification showed a significant decrease in G3 and G4. Zymography indicated an increase in the latent and intermediate isoforms $(72 \mathrm{kDa}$ and $68 \mathrm{kDa}$ respectively) of MMP-2 in the same groups, along with a tissue disorganization and a high cellularity revealed through hematoxylin-eosin stains. Polarization microscopy and birefringence analysis revealed that all experimental groups presented a significant disorganization of collagen fibers when compared to control. Conclusion. In this way we believe the chronic use of AAS leads to tissue disorganization, thus increasing the chance of tendon rupture.
\end{abstract}

KEY WORDS

collagen; metalloproteinase; polarization; Achilles tendon

\section{BACKGROUND}

The use of anabolic-androgenic steroids (AAS) has grown into a worldwide substance abuse problem over the last several decades. Today, the great majority of AAS users are not competitive athletes, but typically young to middleaged men who use these drugs primarily for aesthetic purposes (1).

The doses administered can be 10 to 100 times higher than the therapeutic recommendations, giving rise to hyperandrogenism. Even though these amounts promote increased strength and greater muscle development, a hormonal disorder is developed with a variety of harmful consequences (2).
The side effects of AAS use in both sexes include the increase in strength, libido, acne eruptions, risk of cysts and liver cancer, contribution to the onset of cardiovascular disease, influence over central nervous system functions and the possibility of chronic kidney disease emergence $(3,4,5,6)$. Studies demonstrated that AAS can exhibit adverse effects also on the musculoskeletal system, increasing the risk of tendon rupture since the increase in muscle mass and strength is not followed by the tendinous tissue. It leads to morphological and biomechanical properties alterations that predispose the tissue to microtraumas and ultimately, rupture. It has also been shown that the administration of 
AAS, or its combination with training, reduces the expression of key genes involved in the adaptation of the tendon with collagens I and III $(7,8)$.

A tendon is a unique form of connective tissue that connects the muscle to the bone. This tissue has specific properties that allow it to transmit the force of muscle contraction to the skeleton in order to maintain posture or produce movement (9). The literature shows that the Achilles tendon is the most affected and it is known that the degradation of collagen precedes tendinous rupture in the patients (10). Physical exercise also modifies the structure, mechanical properties, proliferation of fibroblasts, and the synthesis of collagen in human tendons. Previous studies by De Mello Malheiro and colleagues (2009)(11) have shown that Achilles tendons of trained and adapted rats presented an increase in their thickness, had higher cellularity and a greater fraction of the volume of epitenon blood vessels.

AAS should be studied regarding not only its benefits but also analyzing their risks and consequences caused by abusive and unmonitored use. The objective of this study is to assess the effects of nandrolone deacanoate over the components of tendinous tissue, and through them, promote alternatives to minimize or treat the lesions resulting from the steroids overuse.

\section{MATERIALS AND METHODS}

Animal care was in accordance with the European Convention for the Protection of Vertebrate Animals used for Experimental and Other Scientific Purposes and is consistent with the ethical principles of animal experimentation adopted by the Brazilian College of Animal Experimentation (COBEA). The present study was approved by the Committee on Ethics in Animal Use (CEUA) of UNIFALMG (Protocol No. 588/2014). The research was conducted ethically according to international standards and as required by the journal (12).

\section{Experimental groups}

In this study, 20 male Wistar rats were used, with five animals per group per analysis, with a mean age of 60 days and weight ranging from 300 to $350 \mathrm{~g}$. The rats were housed two per cage in a 12 -h light-dark cycle at $23^{\circ} \mathrm{C}$, with free access to commercial food and water. The animals were distributed in the following groups:

G1 - control group (sedentary animals).

G2 - sedentary animals treated with nandrolone decanoate (Deca Durabolin $25 \mathrm{mg}$ ) - 1mg.100g-1.

G3 - trained animals.
G4 - trained animals treated with nandrolone decanoate (Deca Durabolin 25 mg) - 1mg.100g-1.

\section{Treatment}

The anabolic steroid nandrolone decanoate (Deca Durabolin $25 \mathrm{mg}$ ) was administered by a single intramuscular injection into the hind limb of the animals once a week for 8 weeks (13).

The training sessions (swimming with progressive loading) happened at the same time and was started with progressive frequency and increasing overload up to $5 \%$ of the weight of the animals, increasing the effort of the mice during swimming (13).

First week: three weekly sessions, of 10 minutes each, on alternate days, without load.

Second week: four weekly sessions of 20 minutes each, with a load of $2.5 \%$ of the animal's body weight. From the third week: five weekly sessions of 40 minutes each, with a load of $5 \%$ of the animal's body weight.

To obtain training overloads, lead weights equivalent to $5 \%$ of the body mass, placed immediately before swimming by means of an elastic strap attached to the thorax, was used, so as not to impair the dynamics of the movement. During swimming, the animals were monitored so they do not float or lean on the edge of the tank and stop moving. The weight of the animals was checked weekly for the correction of the load value (13). After the treatment, the animals undergo euthanasia with deepening of anesthesia (Isoflurane), for the removal of the Achilles tendon.

\section{BIOCHEMICAL ANALYSIS}

\section{Extraction procedures}

The calcaneal tendon was removed and treated with 25 volumes of $4 \mathrm{M}$ guanidine hydrochloride $(\mathrm{GuHCl})$ containing $20 \mathrm{mM}$ EDTA and $1 \mathrm{mM}$ PMSF in $50 \mathrm{mM}$ sodium acetate buffer $\mathrm{pH} 5.8$ for $24 \mathrm{~h}$ at $4^{\circ} \mathrm{C}$ with stirring (14). Afterward, the material was centrifuged $(13,000 \times \mathrm{g}, 25 \mathrm{~min}$, $4^{\circ} \mathrm{C}$ ), and the supernatant was used for non- collagenous protein dosage.

\section{Quantification of proteins}

Samples of the extracts of each experimental group were used. Non-collagenous proteins were quantified according to the Bradford method (15) using bovine serum albumin as standard. The absorbance was measured at 595 nm. 


\section{Zymography}

Metalloproteinase analyses were performed according to the methods of zymograpphy according to Marquetti and colleagues (2006) ${ }^{(16)}$. The samples were incubated in extraction buffer (50mM Tris- $\mathrm{HCl} \mathrm{pH} 7.4,0.2 \mathrm{M} \mathrm{NaCl}$, $0.1 \%$ Triton $\mathrm{X}-100,10 \mathrm{mM} \mathrm{CaCl} 2$, and protease inhibitor $100 \mu \mathrm{L} / 10 \mathrm{~mL}$ ) at $4{ }^{\circ} \mathrm{C}$ for $24 \mathrm{~h}$. In total, $20 \mu \mathrm{g}$ of protein were loaded in each well. Polyacrylamide gels prepared with $2 \mathrm{mg} / \mathrm{mL}$ of gelatin. Then, the gels were incubated in incubation buffer $(50 \mathrm{mM}$ Tris- $\mathrm{HCl} \mathrm{pH} 8.4,5 \mathrm{mM} \mathrm{CaCl}$, and $1 \mu \mathrm{M} \mathrm{ZnCl2}$ ) overnight at $37{ }^{\circ} \mathrm{C}$. The gels were stained with Coomassie Brilliant Blue R-250 and bleached $(30 \%$ methanol and 10\% acetic acid in water). Finally, the gels were placed in shrinking solution $30 \%$ methanol and $3 \%$ glycerol).

\section{Morphological and birefringence analysis and measurements}

The tendons were fixed using a $4 \%$ formaldehyde solution in Millonig's buffer $(0.13 \mathrm{M}$ sodium phosphate, 0.1 $\mathrm{M} \mathrm{NaOH}-\mathrm{pH} 7.4$ ) for $24 \mathrm{~h}$ at $4^{\circ} \mathrm{C}$ and washed in water, ethanol dehydrated, diaphanized with xylene and paraffin-embedded. Longitudinal serial sections of $7 \mu \mathrm{m}$ were stained with hematoxylin-eosin ( $\mathrm{HE}$ ) and analyzed under an Olympus BX 60 light microscope. Birefringence properties were studied using an Olympus BX51-P BX2 polarizing microscope and an image analyzer (Image-Pro Plus 6.3, Media Cybernetics, Inc.-Silver Spring, MD, USA). Since birefringence appears visually as brilliance, this phenomenon was measured with the image analyzer and expressed as gray average (GA) values in pixels, after its calibration ( 8 bits $=1$ pixel). The major tendon axis was positioned at $45^{\circ}$ to the crossed analyzer and polarizer during the measurements. Considering that collagen bundles exhibit two kinds of birefringences: intrinsic birefringence (Bi) and form or textural birefringence $(\mathrm{Bf})$, total birefringence (sum of Bi and Bf) was used in this study. The measurements the tendons in each experimental group were made after immersing the sections in water (17).

\section{Statistical analyses}

All results were expressed as the mean \pm standard deviation. For biochemical analysis, data from different experimental groups were analyzed by analysis of variance (oneway ANOVA) followed by the Tukey test. The level of significance was $\mathrm{p}<0.05$. The Mann- Whitney test was used only for analysis of the birefringence measurements. The analysis was carried out in GraphPad Prism® (GraphPad Software, La Jolla, CA, USA).

\section{RESULTS}

After 8 weeks of exercise and combined treatment with AAS, the animals were euthanized and the tendons were submitted to morphological and biochemical analysis, generating the results now described.

The sections containing the tendons were stained using Hematoxylin-eosin (Figure 1). G1 (Figure 1-A) presents ordinary tendon morphology, including peritendinous sheath. Epitenon thickness (black bar) was evident in all experimental groups, especially in G2 (Figure 1-B). Also, it was possible to observe a higher cellularity and tissue disorganization in G2, G3, and G4 (Figure 1-B, C, D, E, and F). In the polarization microscopy, the organization degree of the collagen fibers is observable as an increase in the brightness of the section. The images (Figure 2) reveal that the control group (G1) presented greater brightness than all the others. When we observe the G2 group, we note that the nandrolone decanoate alone was enough to change the organization degree in the tendon tissue. Also, the exercise caused similar effects without the influence of other variables (G3). Birefringence analysis (Table I) confirms that all treatment groups were statistically inferior to control, G1. From the values, it is also possible to observe that G2 and G3 did not present difference from each other. On the other hand, the combination of exercise and AAS (G4) caused the greatest disorganization observed in all the experiments.

A zymography of the processed tendon was performed to detect gelatinases. Three isoforms of MMP-2 could be observed: latent, $72 \mathrm{kDa}$; intermediate, $68 \mathrm{kDa}$ and active, $62 \mathrm{kDa}$. Furthermore, no other gelatinases were evident in the gels (Figure 3).

Band densitometry was used to quantify the band's brightness, the values were submitted to ANOVA. It revealed that for latent and intermediate isoforms of MMP-2, G4 was higher than the other groups. The intermediate MMP-2 in G3 was also higher than the control group.

Quantification of non-collagenous proteins (NCP) demonstrated a significant decrease in groups G3 and G4 when compared to the control, G1 (Figure 4).

\section{DISCUSSION}

The aim of this study was to understand the effects of the chronic use of nandrolone decanoate, an anabolic agent, and exercise on extracellular matrix components of the 

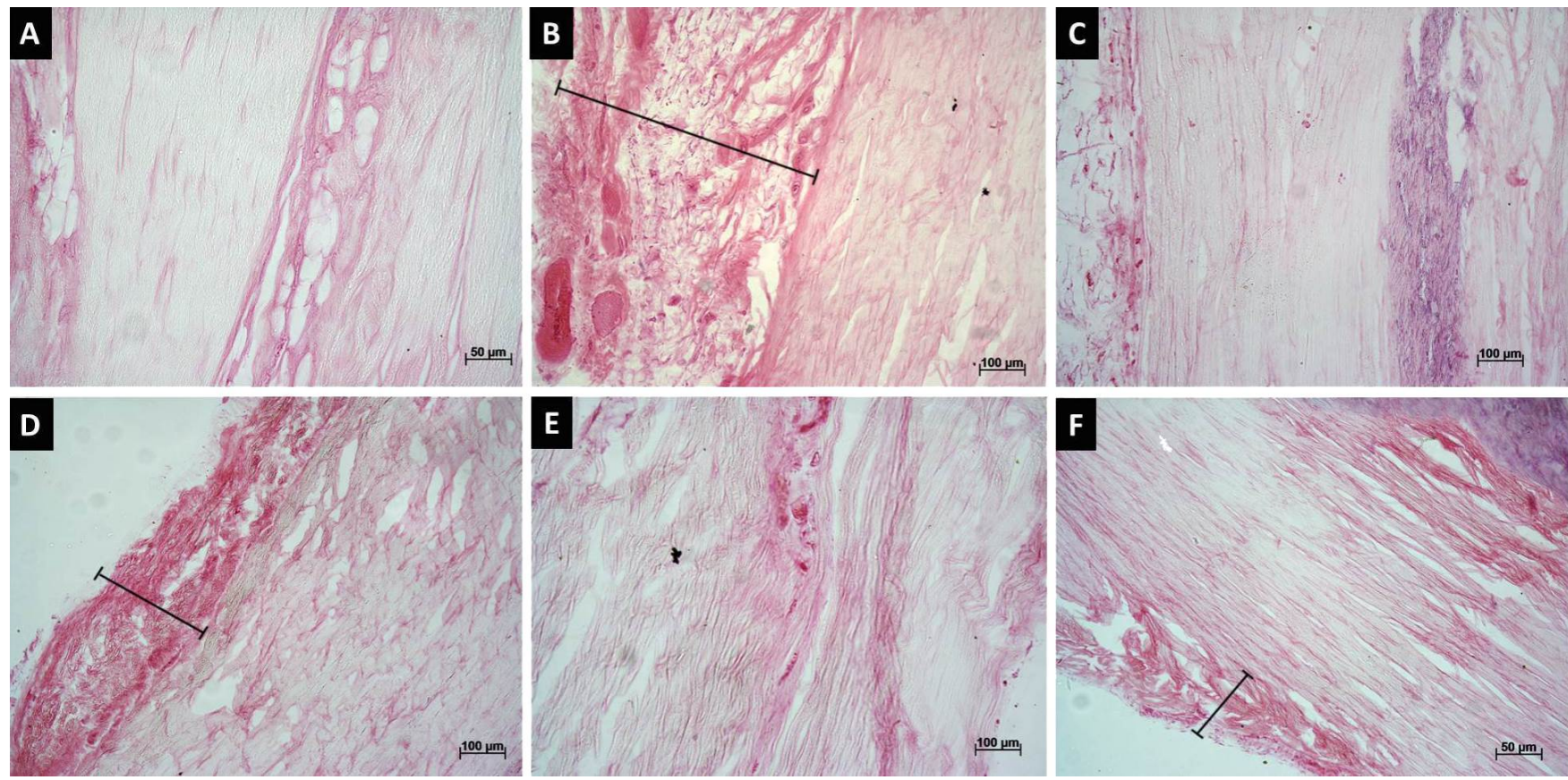

Figure 1. Longitudinal sections of tendons of the different experimental groups. A: G1, B and C: G2, D and E: G3, F: G4. Notice in $B$ the thickness of the epitenon. In D, E and F, observe tissue disorganization and higher cellularity when compared to G1.
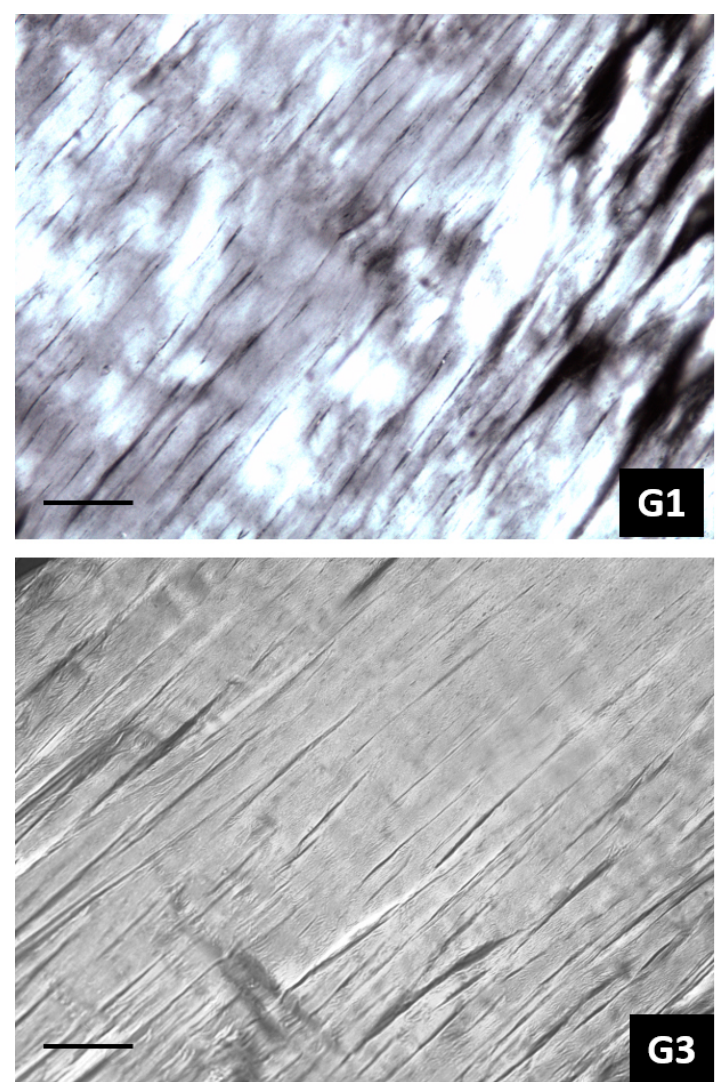
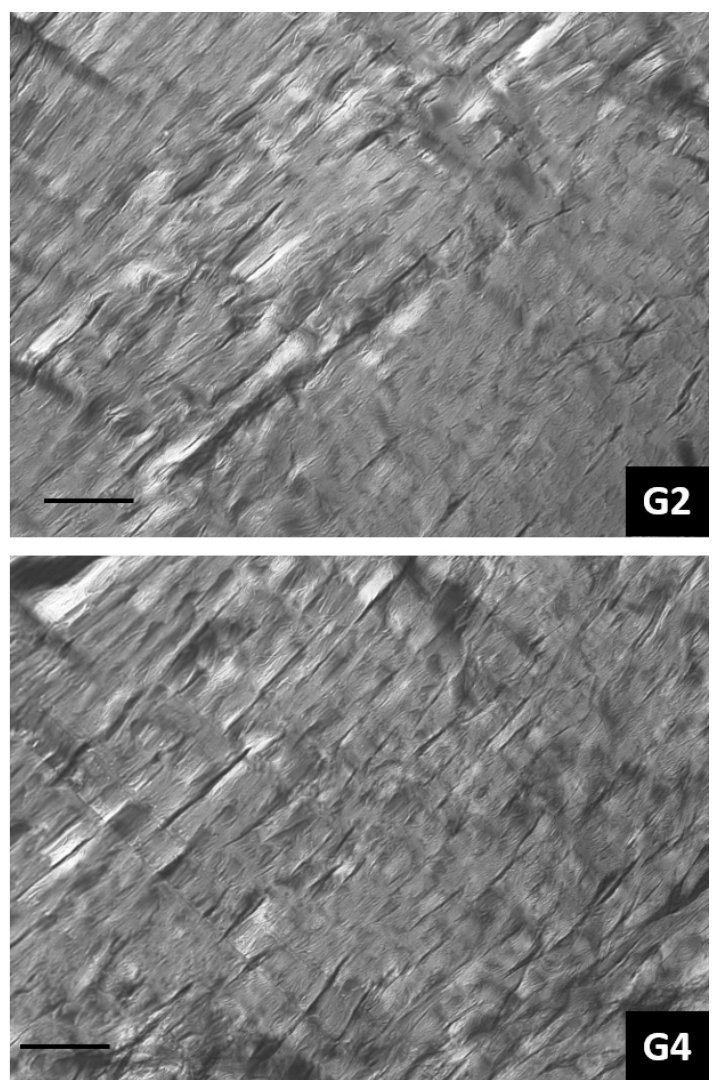

Figure 2.

Longitudinal

sections of tendons

of the different

experimental

groups observed

in polarization microscopy. For birefringence analysis the largest tendon axis was positioned at $45^{\circ}$ to the polarizers. Note the presence of higher brightness in G1 when compared to the other groups, indicating the high organization of the collagen fibers in the whole tissue. Bar: $40 \mu \mathrm{m}$. 
Table I. Birrefringence Analysis

\begin{tabular}{llll}
\hline Groups & $\begin{array}{l}\text { TR (Average shades of } \\
\text { gray) }\end{array}$ & Comparisons & Mann-Whitney Test (p) \\
\hline G1 & 211,53 & $*$ & 0,0001 \\
\hline G2 & 146,07 & G2 $\times$ G3 & 0,1413 \\
\hline G3 & 149,66 & G3 5 G4 & 0,0001 \\
\hline G4 & 130,04 & G2 $\times$ G4 * & 0,0001 \\
\hline
\end{tabular}

The long axis of the tendon was positioned at $45^{\circ}$ to the crossed polarizers. The number of measurements (100) was performed randomly on 12 tendon cuts in each group. TR: Transection region.

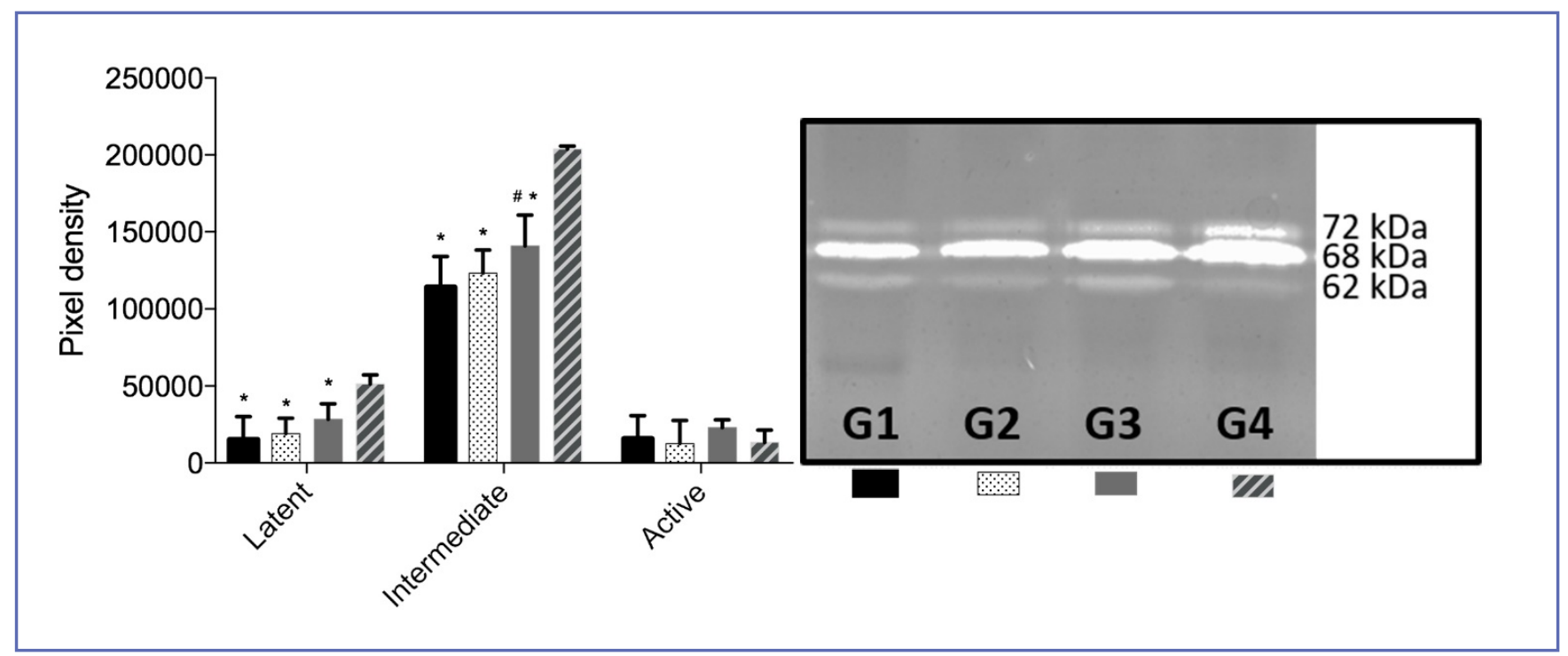

Figure 3. Zymography and band densitometry results for gelatinases of rat tendon extract, from the different experimental groups. Observe the presence of latent $(72 \mathrm{kDa})$, intermediate $(68 \mathrm{kDa})$ and active $(62 \mathrm{kDa})$ isoforms in all groups analyzed. It should be noted that the G1 group presents faint bands in comparison to the other groups. The G4 group shows the latent and intermediate isoforms bands that are more marked when compared to the other groups. Other MMPs were not detected. (\# different from G1; * different from G4).

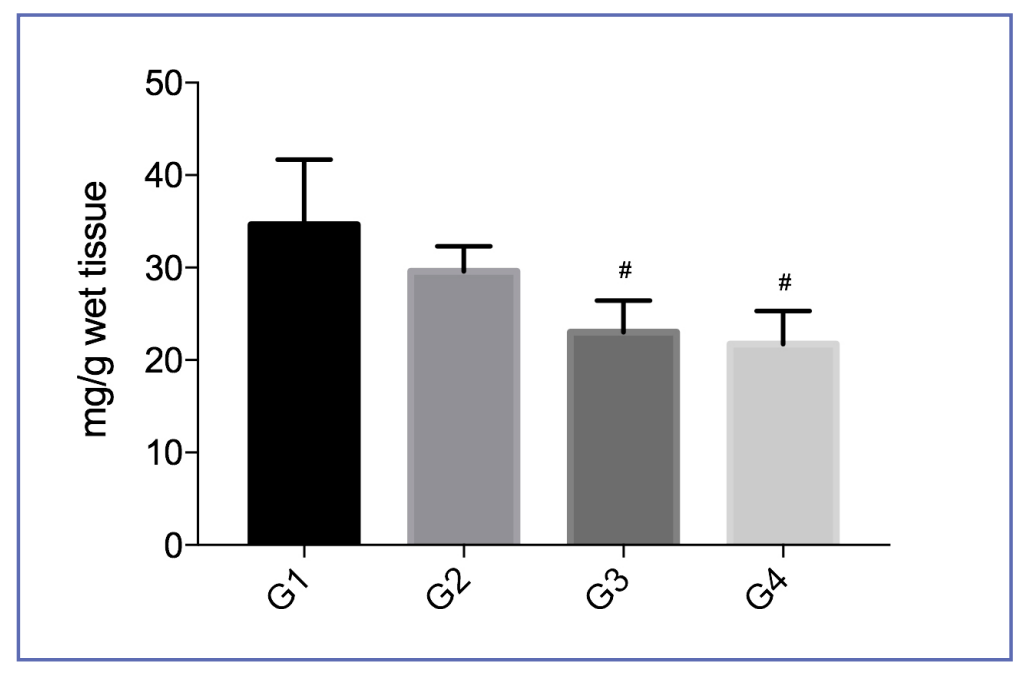

Figure 4. Concentration of non-collagenous proteins ( $\mathrm{mg} / \mathrm{g}$ wet tissue) in the different groups. Observe that G3 and G4 had significant lower amount of non-collagenous proteins than $\mathrm{G} 1$; $p<0.05$. (\# different from G1). 
tendons. The information obtained should help future studies to promote alternatives to minimize or treat the lesions resulting from the steroids overuse.

As stated in the results section, the exercise alone was enough to change some tendon characteristics, including tissue organization, protein content and metalloproteinase profile. Other researchers have already found similar results including different types of exercises $(11,16,18)$.

Other researchers obtained similar founds regarding the epitenon $(11,16)$. Exercises can augment tendon metabolism, with an increase in blood flow (19) and vascularization $(7,11)$. Together, these factors can naturally cause swelling in the area, due to a rise in water content and extracellular matrix components. Despite this increase, there was no evidence of inflammatory infiltrate in the samples, which may account for the lack of MMP-9.

Depending on the type of exercise which the animals are submitted, different metalloproteinase profiles can emerge. Researchers found only MMP-2 evidence after exercising animals, with (11) or without (20) inflammatory infiltrate, while Marqueti and colleagues (2006) (16) also found only MMP-2 evidence in gel, even with the presence of marked inflammatory infiltrate. However, Koskinen and colleagues (2004) (18) found high MMP-9 values after exercise in humans.

The use of nandrolone decanoate without exercise association (G2) resulted in collagen fiber disorganization similar to the one found in G3. On the other hand, only G3 presented a significative reduction of NCP which was not observed in G2. The combination of exercise and AAS use (G4), produced more significant changes in the tissue, regarding collagen organization, and MMP concentration. Interestingly, the change in the epitenon thickness was not as marked as when the animals received only the AAS. Marqueti and colleagues (2014) (7) reported that the AAS use diminished the tendon adaptations to exercises, including reduction of vasculogenesis. The association of muscle hypertrophy and prevention of tendon adaptation to workload can be a source of tendon injury in either athletes or non-athletes users. The excessive amount of MMPs found are especially important considering that degradation of collagen precedes tendinous rupture in the patients (10).

\section{REFERENCES}

1. Kanayama G, Pope Jr HG. History and epidemiology of anabolic androgens in athletes and non-athletes. Mol. Cell. Endocrinol. 2017 https://doi.org/10.1016/j.mce.2017.02.039

2. Smurawa TM, Congeni JA. Testosterone precursors: use and abuse in pediatric athletes. Pediatr. Clin. North Am. 2007; 54 : 787-96, xii.
This study showed that there was a large decrease in the non-collagenous proteins in G3 and G4. This loss of proteins causes damages to the tendon because they are the components that give it structure, and such reduction may lead to an increase in the chance of rupture.

Collagen degradation is initiated extracellularly by MMPs, mostly MMP-2 and MMP-9. Under normal conditions, MMPs are present in low concentrations. After tissue damage, proteolysis is required, to remove the damaged matrix and aid in the synthesis of new tissue, an increase in MMPs indicates that the matrix is undergoing tissue degradation and repair $(16,21)$.

When inflammatory processes occur, the collagen fibers disorganize, the inflammatory cells are directed to the location of the lesion and there is the activation of enzymes, like MMPs, that degrade the GAGs and proteins. Tendon biomechanics are compromised due to disorganization of collagen fibers, and tendons are more susceptible to ruptures during healing (22).

These reports indicate that the combination of nandrolone decanoate and exercise induces noxious changes in tendons and there is a greater predisposition to rupture. These changes in tendons occur mainly due to the disorganization of the collagen fibers. Even the AAS being used without association with exercise increases the stiffness of the tendon, decreases the elasticity and the amount of energy it can absorb (7).

In summary, our results showed that the Achilles tendon suffers a high degree of tissue disorganization with the combination of exercise and the AAS nandrolone decanoate, with a high level of collagen bundles disorganization, a decrease in levels of non-collagenous proteins, and an increase in MMP-2, indicating a remodeling event taking place. This data leads us to believe that chronic use of the AAS associated with exercise can promote significant injury in the tissue that may promote tendon rupture.

\section{Conflict of Interest}

The authors declare that they have no conflict of interest (23)

3. Freitas AC de, Damião B, Alves DM, et al. Efeitos dos anabolizantes sobre a densidade de neurônios dos núcleos da base. Rev. Bras. Med. do Esporte 2017; 23: 213-216.

4. Solimini R, Rotolo MC, Mastrobattista L. Hepatotoxicity associated with illicit use of anabolic androgenic steroids in doping. Eur. Rev. Med. Pharmacol. Sci. 2017; 21: 7-16. 
5. van Amsterdam J, Opperhuizen A, Hartgens F. Adverse health effects of anabolic-androgenic steroids. Regul. Toxicol. Pharmacol. 2010; 57: 117-123.

6. Westlye LT, Kaufmann T, Alnæs D, et al. Brain connectivity aberrations in anabolic-androgenic steroid users. Neuroimage (Amst). 2017; 13: 62-69.

7. Marqueti RC, Paulino MG, Fernandes MN, et al. Tendon structural adaptations to load exercise are inhibited by anabolic androgenic steroids. Scand. J. Med. Sci. Sports 2014; 24: e39-e51.

8. Marqueti RC, Heinemeier KM, Durigan JL, et al. Gene expression in distinct regions of rat tendons in response to jump training combined with anabolic androgenic steroid administration. Eur. J. Appl. Physiol. 2012; 112: 1505-1515.

9. Nourissat G, Berenbaum F, Duprez D. Tendon injury: from biology to tendon repair. Nat. Rev. Rheumatol. 2015; 11 : 223-233.

10. Gulati V, Jaggard M, Al-Nammari SS, et al. Management of achilles tendon injury: A current concepts systematic review. World. J. Orthop. 2015; 6: 380-386.

11. De Mello Malheiro OC, Giacomini CT, Justulin Jr. LA, et al. Calcaneal tendon regions exhibit different MMP-2 activation after vertical jumping and treadmill running. Anat Rec 2009; 292: 1656-1662.

12. Padulo J, Oliva F, Frizziero A, et al. Muscles, Ligaments and Tendons Journal - Basic principles and recommendations in clinical and field Science Research: 2016 Update. MLTJ 2016; 6(1): $1-5$.

13. Frankenfeld SP, de Oliveira LP, Ignacio DL, et al. Nandrolone decanoate inhibits gluconeogenesis and decreases fasting glucose in Wistar male rats. J. Endocrinol. 2014; 220: $143-153$

14. Heinegård D, Sommarin Y. Isolation and characterization of proteoglycans. Meth Enzymol. 1987; 144:319-72.
15. Bradford MM. A rapid and sensitive method for the quantitation of microgram quantities of protein utilizing the principle of protein-dye binding. Anal. Biochem. 1976; 72: 248-254.

16. Marqueti RC, Parizotto NA, Chriguer RS, et al. Androgenic-anabolic steroids associated with mechanical loading inhibit matrix metallopeptidase activity and affect the remodeling of the achilles tendon in rats. Am. J. Sports Med. 2006; 34: 1274-1280.

17. Vidal B de C, Mello MLS. Optical anisotropy of collagen fibers of rat calcaneal tendons: An approach to spatially resolved supramolecular organization. Acta Histochem. 2010; 112: 53-61.

18. Koskinen SO, Heinemeier KM, Olesen JL, et al. Physical exercise can influence local levels of matrix metalloproteinases and their inhibitors in tendon-related connective tissue. J. Appl. Physiol. 2004; 96: 861-864.

19. Magnusson SP, Hansen P, Kjær M. Tendon properties in relation to muscular activity and physical training. Scand. J. Med. Sci. Sports. 2003; 13: 211-223.

20. Huisman E, Lu A, Jamil S, Mousavizadeh R, McCormack R, Roberts C, Scott A. Influence of repetitive mechanical loading on MMP2 activity in tendon fibroblasts. J. Orthop. Res. 2016; 34: 1991-2000.

21. Guerra FD, Vieira CP, Almeida MS, et al. Pulsed LLLT improves tendon healing in rats: a biochemical, organizational, and functional evaluation. Lasers Med. Sci. 2014; 29: 805-811.

22. Vieira CP, Guerra FD, Oliveira LP, Almeida MS, Marcondes MC, Pimentell ER.. Green tea and glycine aid in the recovery of tendinitis of the Achilles tendon of rats. Connect. Tissue Res. 2015; 56: 50-58.

23. Padulo J, Oliva F, Frizziero A, Maffulli N. Muscles, Ligaments and Tendons Journal - Basic principles and recommendations in clinical and field Science Research: 2018 update. MLTJ 2018; 8(3): $305-307$. 